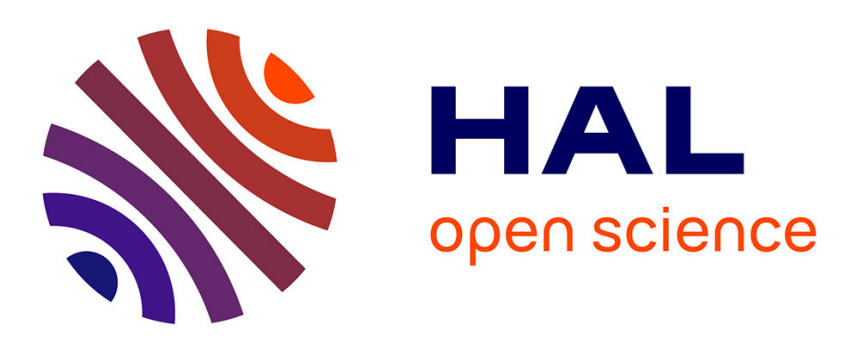

\title{
Rapid and automatic discrimination between facial expressions in the human brain
}

Fanny Poncet, Jean Yves Baudouin, Milena Dzhelyova, Bruno Rossion, Arnaud Leleu

\section{- To cite this version:}

Fanny Poncet, Jean Yves Baudouin, Milena Dzhelyova, Bruno Rossion, Arnaud Leleu. Rapid and automatic discrimination between facial expressions in the human brain. Neuropsychologia, 2019, 129, pp.47-55. 10.1016/j.neuropsychologia.2019.03.006 . hal-02406196

\section{HAL Id: hal-02406196 https://hal.science/hal-02406196}

Submitted on 22 Oct 2021

HAL is a multi-disciplinary open access archive for the deposit and dissemination of scientific research documents, whether they are published or not. The documents may come from teaching and research institutions in France or abroad, or from public or private research centers.
L'archive ouverte pluridisciplinaire HAL, est destinée au dépôt et à la diffusion de documents scientifiques de niveau recherche, publiés ou non, émanant des établissements d'enseignement et de recherche français ou étrangers, des laboratoires publics ou privés.

\section{(ㄷ)(1) $\$$}

Distributed under a Creative Commons Attribution - NonCommercial| 4.0 International 


\title{
Rapid and automatic discrimination between facial expressions in the human brain
}

\author{
Fanny Poncet ${ }^{1}$, Jean-Yves Baudouin ${ }^{1,2}$, Milena P Dzhelyova ${ }^{3}$, Bruno Rossion $^{3,4,5}$, Arnaud Leleu ${ }^{1}$ \\ ${ }^{1}$ Developmental Ethology and Cognitive Psychology group, Centre des Sciences du Goût et de \\ I'Alimentation, CNRS, Université Bourgogne Franche-Comté, Inra, AgroSup Dijon, F-21000 Dijon, \\ France \\ ${ }^{2}$ Laboratoire Développement, Individu, Processus, Handicap, Éducation (DIPHE), Département \\ Psychologie du Développement, de l'Éducation et des Vulnérabilités (PsyDÉV), Institut de \\ psychologie, Université de Lyon (Lumière Lyon 2), 69676 Bron cedex, France \\ ${ }^{3}$ Psychological Sciences Research Institute, Institute of Neuroscience, University of Louvain (UCL), \\ 1348 Louvain-la-Neuve, Belgium \\ ${ }^{4}$ Université de Lorraine, CNRS, CRAN - UMR 7039, F-54000 Nancy, France \\ ${ }^{5}$ Université de Lorraine, CHRU-Nancy, Service de Neurologie, F-54000, France
}

Corresponding authors: FP (Fanny.Poncet@u-bourgogne.fr) and AL (Arnaud.Leleu@u-bourgogne.fr)

Corresponding address : Équipe Éthologie développementale et Psychologie cognitive, Centre des Sciences du Goût et de l'Alimentation, $9^{\mathrm{E}}$ Boulevard Jeanne d'Arc, 21000 Dijon, France 


\section{Abstract}

Automatic responses to brief expression changes from a neutral face have been recently isolated in the human brain using fast periodic visual stimulation (FPVS) coupled with scalp electroencephalography (EEG). Based on these observations, here we isolate specific neural signatures for the rapid categorization of each of 5 basic expressions, i.e., when they are directly discriminated from all other facial expressions. Scalp EEG was recorded in 15 participants presented with pictures alternating at a rapid $6 \mathrm{~Hz}$ rate (i.e., one fixation/face, backward- and forward-masked). In different stimulation sequences, an expressive (angry, disgusted, happy, fearful, or sad) or a neutral face arose every 5 pictures (i.e., at $6 / 5=1.2 \mathrm{~Hz}$ ), among pictures of the same individual expressing the other emotions randomly. Frequency-domain analysis indicated a robust (i.e., recorded in every individual participant) and objective (i.e., at the predefined $1.2 \mathrm{~Hz}$ frequency and its harmonics) expression-specific brain response over occipito-temporal sites for each emotion and neutrality. In this context of variable expressions, while neural responses to the different expressions (Anger, Disgust, Happiness, Sadness) were dissimilar qualitatively, a much larger specific signature for neutral faces as compared to facial expressions was found. Interestingly, Fear also elicited a strong contrasted response with other facial expressions, associated with a specific neural signature over ventral occipito-temporal sites. Collectively, these findings reveal that specific EEG signatures for different facial expressions can be isolated in the human brain, pointing to partially different neural substrates. In addition, they provide support for a strong and highly selective neural response to fear at the system-level, in line with the importance of this emotional expression for biological survival.

Key-words: Fast Periodic Visual Stimulation, EEG, frequency-tagging, facial expression, discrimination, categorization. 


\section{Introduction}

Quickly recognizing expressive facial cues about internal emotional states of others is a crucial ability in the human species, especially for adapted social interactions. Despite the wide variety of expressions, some of them have been described as universal basic emotions, being recognized by allocating distinct patterns of facial actions to discrete emotion categories (Darwin, 1872; Ekman, 1993; for meta-analyses, see Elfenbein \& Ambady, 2002; Sauter \& Eisner, 2013). More specifically, according to Ekman's proposal (Ekman, 1992, 1994; Ekman \& Friesen, 1971; Ekman, Friesen, \& Ellsworth, 1972), facial expressions are perceived as belonging to qualitatively discrete emotional categories with sharp, clear-cut boundaries between each category (although see Barrett \& Wager, 2006; Russell, 1980; Woodworth \& Schlosberg, 1954).

At the neural level, isolating a specific signature for each facial expression has been rather challenging. A number of studies have shown that the processing of every facial expression elicits activity in face-selective regions of the inferior occipital gyrus (IOG), lateral fusiform gyrus (LatFG) and posterior superior temporal sulcus (pSTS) (Calder \& Young, 2005; Haxby, Hoffman, Gobbini, 2000). The latter region is known to play an important role in processing dynamic changes in expression (Puce et al., 2003; Srinivasan et al., 2016), although significant responses to facial expressions have also been recorded in the LatFG and the ventral face processing system in general (e.g., Kawasaki et al., 2012). Outputs from the pSTS are thought to project to an extended faceprocessing network, including the amygdala, the insula, and the limbic system, for further analysis of facial expression. At this level, distinct and discrete brain regions are considered to be involved in the processing of the different basic facial emotion categories; namely happiness, anger, disgust, fear, and sadness (e.g., Vytal, \& Hamann, 2010 for a meta-analysis). However, the specificity of these regions for processing various expressions has been questioned (Barrett \& Wager, 2006; Barrett, Lindquist, Bliss-Moreau, Duncan, \& Brennan, 2007). In addition, recent studies reported that emotional facial expressions also elicit separable patterns of brain activity in regions responding to all facial expressions, such as the anterior and posterior STS and the amygdala (Harris, Young, \& Andrews, 2012, 2014; Said, Moore, Engell, Todorov, \& Haxby, 2010; Whalen et al., 2013). In sum, neuroimaging studies have struggled to identify consistent specific patterns of neural activation for each major facial expression category.

With scalp electroencephalography (EEG), a technique that is not only advantageous for its high temporal resolution but also for being able to measure direct brain activity in various populations (e.g., neurotypical adults, children and infants, as well as neurological or psychiatric patients), specific neural signatures for some facial expressions have also been very difficult to isolate 
when using standard event-related potentials (ERPs) approaches (for reviews, see Calvo \& Nummenmaa, 2016; Vuilleumier \& Pourtois, 2007). The differential processing of emotional vs. neutral faces is thought to primarily occur around $150-300 \mathrm{~ms}$ post-stimulus onset over occipitotemporal and occipito-parietal sites (e.g., Calvo, Marrero, \& Beltrán, 2013; Leleu, Godard, Dollion, Durand, Schaal, \& Baudouin, 2015; Williams, Palmer, Liddell, Song, \& Gordon, 2006). The categorization of facial expressions in discrete categories is further considered from about $300 \mathrm{~ms}$ after stimulus-onset, at the level of several late components with distinct topographies across studies (P3b: Luo, Feng, He, Wang, \& Luo, 2010; late positive potential/LPP: Leppänen, Kauppinen, Peltola, \& Hietanen, 2007; slow positive waves/SPWs: Calvo \& Beltrán, 2013; posterior negativity: Ashley, Vuilleumier, \& Swick, 2004). Conclusions about brain facial expressions categorization remain fragile however, and no solid and systematic pattern has been reliably found for each emotion category (for discussions, see Calvo \& Nummenmaa, 2016; Dzhelyova et al., 2017). This is not surprising since ERPs are generally obtained by contrasting the sudden onset of a facial expression stimulus to a uniform visual field (i.e., background). The recorded brain response thus largely reflects the general visual processing of the stimulus (including specific responses to faces or to all expressive faces), making it difficult to isolate subtle differential neurophysiological activities across expressions.

To tackle this issue, recent studies have used Fast Periodic Visual Stimulation (FPVS) associated with EEG recordings to show robust neural responses to brief expression changes directly contrasted with neutral faces (Dzhelyova, Jacques \& Rossion, 2017; Leleu, Dzhelyova, Rossion, Brochard, Durand, Schaal \& Baudouin, 2018). The FPVS-EEG approach relies on the brain's property to synchronize its activity with stimuli displayed periodically (Adrian \& Matthews, 1934), eliciting EEG responses at the same frequency (Regan, 1989; Norcia, Appelbaum, Ales, Cottereau, \& Rossion, 2015 for review). FPVS-EEG has high objectivity because responses are measured at a predetermined frequency of stimulation. This response can have a high signal-to-noise ratio (SNR), even within a few minutes of recording. By using a rapid base rate of visual stimulation and introducing a systematic stimulus change at a lower periodic rate, FPVS-EEG designs allow dissociating two distinct brain responses elicited at two different frequencies within a single stimulation sequence (Braddick, Wattam-Bell \& Atkinson, 1986; Liu-Shuang, Norcia \& Rossion, 2014). The first response, tagged at the base frequency, reflects the neural processing of all visual events occurring at each stimulus-onset, a mixture of both low- and higher-level processes. Interestingly, the second response, tagged at the lower frequency, is a direct (i.e., without post-hoc subtraction) marker of the differential neural processes specifically elicited by the specific stimulus change in contrast to the base stimuli. In recent years, this approach has been successfully used to investigate the categorization of face stimuli as faces (against various nonface objects; e.g., Rossion et al., 2015), face individuation (Liu-Shuang et 
al., 2014; 2016; Dzhelyova \& Rossion, 2014a, 2014b), and facial expression processing (Dzhelyova et al., 2017; Leleu et al., 2018).

In the studies by Dzhelyova et al. (2017) and Leleu et al. (2018), specific expressions (either, happy, fear, or disgust, with anger and sadness in Leleu et al., 2018) were displayed regularly (at 1.2 or $1.33 \mathrm{~Hz}$ ) in different sequences with the neutral face of the same individual at the base frequency ( 6 or $12 \mathrm{~Hz}$ ), mimicking brief expression changes from neutrality. The face stimuli were displayed either upright or upside-down (Dzhelyova et al., 2017) or with linearly increasing intensities (Leleu et al., 2018). Discrimination of the expressions from the neutral face was indexed by the predefined frequency-tagged brain response to the target expression with: (1) a response to brief changes of facial expression over the right ventral and dorsal occipito-temporal sites, larger for upright than for upside-down faces, and increasing non-linearly with expression intensity; (2) variable amplitudes and topographies for the different expressions, with larger response for disgust than the other expressions, a more dorsal response for happiness, more restricted responses for fear and sadness, a more bilaterally distributed response to fear, and the lowest response for sadness. In both studies, significant responses were evidenced in every individual participant.

Here, we investigate further whether a brain response specifically indexing the categorization of facial expressions, i.e., their assignment into discrete categories from the differential recruitment of brain structures for each facial emotion, can be isolated using FPVS-EEG. Indeed, when contrasting a given facial expression with neutrality, the evoked response may elicit neural activities that are both specific for that expression and common to several if not all expressions. Some brain areas may respond similarly to all expressions, whereas some may respond to a subset or only to a single expression (e.g., Vytal \& Hamann, 2010). Some regions may also respond to all expressions but with separable brain activity (Said et al., 2010). To dissociate the contributions of specific and common neural activities across expressions, we used a design in which variable facial expressions were randomly displayed at a $6 \mathrm{~Hz}$ base stimulation frequency (i.e., 6 stimuli per second, stimulus duration $\approx 167 \mathrm{~ms}$, single fixation limiting eye movements) and directly contrasted to another target expression periodically inserted every $5^{\text {th }}$ stimulus, at a $1.2 \mathrm{~Hz}$ specific frequency (Figure 1). Hence, the brain response tagged at $6 \mathrm{~Hz}$ here reflects both low-level visual processes (e.g., contrast-change) elicited by the rapid stream of stimulation, and higher-level perceptual responses to the rapid change of facial expression. If common neural processes are involved for every facial expression (e.g., neural activities elicited by any facial movement), they are captured by this general visual response to the fast train of facial expressions. In contrast, the brain response tagged at $1.2 \mathrm{~Hz}$ is a direct signature of expression categorization, that is, the differential neural activities elicited for one specific expression against all other possible ones. To determine whether the neutral expression is discriminated from all of the other expressions, it was also included at the $1.2 \mathrm{~Hz}$ expression-specific frequency. We show 
that the brain response to each facial expression can be quantified at the specific $1.2 \mathrm{~Hz}$ frequency and its harmonics (i.e., integer multiples). The expression-specific response is significant in every individual brain, characterized by different scalp topographies according to the emotion expressed, and is larger for the categorization of neutrality and fear, thus reflecting the particular status of these two facial expressions.

\section{Materials and methods}

\subsection{Participants}

Fifteen participants (10 females, mean age $=25.5 \pm 4$ (SD) years, range 20-33 years) were included in the experiment and received financial compensation. All of them reported normal or corrected-to-normal vision and none reported history of psychiatric or neurological disorders. Participants provided written informed consent prior to the experiment and testing was conducted in accordance with the Declaration of Helsinki. It is worth noting that we conducted an exploratory study for which effect size was not pre-experimentally defined and adequate sample size difficult to estimate. We further discuss this point in the Limitations section and provide power analysis conducted on the observed effect size.

\subsection{Stimuli}

Images from 6 individual faces $(01 \mathrm{~F}, 02 \mathrm{~F}, 05 \mathrm{~F}, 07 \mathrm{~F}, 09 \mathrm{~F}, 10 \mathrm{~F})$ were taken from the NimStim database (Tottenham et al., 2009). We selected for each face a neutral expression as well as an expression of anger, disgust, fear, happiness and sadness (Figure 1). Surprise was not used due to its ambiguity in terms of emotional valence (Kim et al., 2004), and the lack of evidence for categorical boundaries between surprise and other expressions (Etcoff \& Magee, 1992). Stimuli were cropped in a medallion-shape window to remove information from the background and hairstyle and presented on a mid-level gray color background (128/255 in grayscale). Images were set to a size of $6 \times 4.8 \mathrm{~cm}$ $\left(6 \times 4.8^{\circ}\right.$ of visual angle at a viewing distance of $\left.57 \mathrm{~cm}\right)$ and equated for mean luminance.

\subsection{Procedure}

The procedure was similar to the experiments of Dzhelyova et al. (2017) and Leleu et al. (2018). Fast periodic visual stimulation (FPVS) was used with a base rate stimulation of $6 \mathrm{~Hz}$ (i.e. 6 images/s), and an expression-specific rate of $1.2 \mathrm{~Hz}$ (same emotion every 5 pictures, see below). Using a 24-inch LED monitor with a $60 \mathrm{~Hz}$ refresh rate and a resolution of $1920 \times 1080$ pixels, stimuli were displayed on a mid-level gray background (128/255 in grayscale) through sinusoidal contrast modulation (0-100 \%) at the base frequency $(6 \mathrm{~Hz})$ with custom Java software (SinStim). At this rapid rate, each stimulus is 
presented for about $167 \mathrm{~ms}$ and reaches full contrast approximately $83 \mathrm{~ms}$ after onset (half a cycle). This rapid mode of stimulation implies that perception occurs at a glance. To avoid low-level repetition effects, the size of the images was randomly varied between $95 \%$ and $105 \%$ (i.e., $5.7 \times$ $4.56^{\circ}$ and $6.3 \times 5.04^{\circ}$ of visual angle respectively) at every stimulation cycle. Sequences presenting one individual face and testing the perception of one specific emotional expression were organized so that one of the emotional expressions is periodically inserted at the expression-specific rate (i.e., $6 / 5=1.2 \mathrm{~Hz}, \approx 833 \mathrm{~ms}$ between two target expressive faces) among the other expressions randomly displayed at the base frequency (Figure 1). As a result, two brain responses reflecting two distinct processes elicited within a single stimulation sequence are projected to different frequencies in the EEG spectrum. EEG amplitude recorded at the $6 \mathrm{~Hz}$ base frequency and its harmonics (i.e., integer multiples of $6 \mathrm{~Hz}: 12 \mathrm{~Hz}, 18 \mathrm{~Hz}$, etc.) reflects a general visual response to the rapid stream of face stimuli changing in contrast, size and expression. In contrast, the neural activity elicited at $1.2 \mathrm{~Hz}$ and harmonics is a direct marker of the automatic categorization of a specific facial expression with no contamination from other processes elicited by any change of facial expression. To determine whether the neutral expression is automatically categorized when directly contrasted against all the other expressions, it was also included at the expression-specific frequency. Hence, the design evaluated the specific response to the five emotional expressions (anger, disgust, fear, happiness and sadness) plus neutrality, for a total of 6 conditions. One advantage of this design is that it directly contrasts every expression category within a single stimulation sequence and thus isolates specific neural responses to one emotional expression against the others.

After EEG-cap placement, participants were seated in a light- and sound-isolated cabin and their head was held on a chinrest at $57 \mathrm{~cm}$ from the screen. Each participant was stimulated with 36 sequences: 6 conditions $\times 6$ individual faces. For each sequence, the stimulation lasted $35 \mathrm{~s}$ on average with a random pre-stimulation interval of 0.5 to $1 \mathrm{~s}$ presenting a blank screen, followed by a gradual fade-in of increasing contrast modulation depth (2.5s), and then by the stimulation (30s). A gradual fade-out lasted $1 \mathrm{~s}$ afterward followed by a random blank post-stimulation interval ( 0.5 to $1 \mathrm{~s})$. The 36 sequences were divided in 6 blocks of 6 sequences, each block presenting one sequence per condition and alternating individual faces. The presentation orders of blocks and sequences within blocks were randomized across participants. The presentation order of the 5 irrelevant expressions at the base frequency followed cycles of the 5 expressions (e.g., anger, disgust, fear, sadness and neutrality when happiness was presented at the expression-specific frequency; Figure 1) whose order was randomized at each cycle. During each sequence, participants were instructed to look at a circle that was always present on the screen and located just below the eyes when the face stimuli were displayed. They had to press the space bar with both index fingers as fast as possible each time the circle changed to a square. These shape-changes briefly (200ms) intervened 5 random times with 
a minimum $2 \mathrm{~s}$ interval between each change. This orthogonal task was used to ensure that participants were attentive. When asked at the end of the experiment, all participants reported having noticed expressive faces during the stimulation, but none detected their periodicity.

\subsection{EEG Acquisition}

Electroencephalographic (EEG) activity was continuously recorded from a BioSemi ActiveTwo amplifier system (BioSemi, The Netherlands) with $64 \mathrm{Ag} / \mathrm{AgCl}$ electrodes located according to the 10-10 classification system. During recording, the Common Mode Sense (CMS) active electrode was used as reference and the Driven Right Leg (DRL) passive electrode was used as ground. Electrode offset was reduced between $\pm 15 \mu \mathrm{V}$ for each channel and EEG was digitalized at a $1024 \mathrm{~Hz}$ sampling rate.

\subsection{EEG preprocessing}

EEG processing was conducted using Letswave 5 (http://nocions. webnode.com/letswave), running on Matlab 2012 (Mathworks, USA), and largely followed analyses steps described in previous studies (Dzhelyova et al., 2017; Leleu et al., 2018). The continuously recorded data were first bandpass filtered at $0.1-100 \mathrm{~Hz}$ (butterworth filter, $4^{\text {th }}$ order) and then downsampled to $256 \mathrm{~Hz}$ to reduce file size and processing time. They were cropped into $35 \mathrm{~s}$-segments for each sequence $11 \mathrm{~s}$ before the fade-in and $0.5 \mathrm{~s}$ after the fade-out). An Independent Component Analysis (ICA) was computed (e.g., Makeig et al., 1996) and components corresponding to eye blinks (recorded over Fp electrodes) and artifacts recorded over frontal and temporal electrodes were removed. Remaining noisy or artifact-ridden electrodes (i.e., with amplitude exceeding $\pm 100 \mu \mathrm{V}$ ) were rebuilt using linear interpolation from the four nearest channels (mean number across participants: 0.4 channels, range: 0-2). All EEG epochs were finally re-referenced to a common average reference.

\subsection{EEG frequency-domain analysis}

Preprocessed data segments were cropped down into 30-sec epochs beginning just after the fade-in and corresponding exactly to thirty-six $1.2 \mathrm{~Hz}$ cycles (for a total of 7680 time bins). The 6 segments corresponding to the 6 sequences for a specific emotion (i.e., 6 individual faces) were then averaged in the time domain for all participants to reduce EEG activity non-phase-locked to the stimuli. The resulting 6 averaged segments (i.e., all conditions) were finally transformed with fast Fourier transform (FFT) and amplitude spectra were extracted for all channels with a high frequency resolution of $1 / 30=0.033 \mathrm{~Hz}$. Individual FFT data were grand-averaged across participants for group analysis. 
To determine the significant harmonics for both general visual (i.e., elicited by the rapid $6 \mathrm{~Hz}$ base stimulation frequency) and expression-specific (i.e., elicited by the $1.2 \mathrm{~Hz}$ periodic presentation of a given facial expression) responses, FFT grand-averaged data were pooled across electrodes and conditions and Z-scores were calculated at each frequency bin by subtracting the mean surrounding noise amplitude (i.e., estimated from the 20 surrounding frequency bins excluding the 2 immediately neighboring and the 2 most extreme values, e.g. Dzhelyova et al., 2017; Leleu et al., 2018) and dividing by its standard deviation. Harmonics were considered significant until Z-scores were no longer above 1.64 ( $p<.05$, one-tailed, signal $>$ noise) for two consecutive harmonics. For the general visual response, significant harmonics were found until the $8^{\text {th }}$ harmonic (i.e., $48 \mathrm{~Hz}$, harmonics were not considered after the $50 \mathrm{~Hz}$ response elicited by $\mathrm{AC}$ power). For the expression-specific response, significant harmonics were found until the $7^{\text {th }}$ harmonic (i.e., $8.4 \mathrm{~Hz}$ ).

Z-scores were then used as a data-driven whole-scalp approach to determine different regions-of-interest (ROIs) to include in statistical analyses, separately for each brain response. FFT data were first grand-averaged across conditions and significant harmonics for each response were summed for each electrode (for the expression-specific response, the $5^{\text {th }}$ harmonic corresponding to the base frequency (i.e., $6 \mathrm{~Hz}$ ) was excluded). Summed harmonics were used to quantify the overall responses in single values (Retter \& Rossion, 2016). Topographical differences were then normalized according to the scalp-wide global power of each brain response (McCarthy \& Wood, 1985). Normalization allows identifying the electrodes over which the response is largest irrespective of its global magnitude across the scalp (Leleu et al., 2018). Z-scores were calculated and the significant electrodes were finally pooled together in three distinct regions of interest (ROIs) considered for further statistical analyses (i.e., different ROIs and analyses for each brain response). For the expression-specific response, ROIs comprised medial occipital (mO: channels $\mathrm{Oz}, \mathrm{lz}, \mathrm{O} 1, \mathrm{O} 2$ ), and lateral (right and left) occipito-temporal (rOT: channels P4, P8, P10, PO4, PO8, IOT: channels P3, P7, $\mathrm{P9}, \mathrm{PO} 3, \mathrm{PO} 7)$ sites. Note that the left-hemispheric channels P3 and PO3 were not significant but included in the IOT ROI to match the significant channels identified in the right hemisphere (i.e., P4 and $\mathrm{PO} 4)$. For the general visual response, the $\mathrm{mO}$ region included 5 channels $(\mathrm{Oz}, \mathrm{lz}, \mathrm{O} 1, \mathrm{O} 2, \mathrm{POz})$, and each lateral ROI included 3 channels (rOT: PO4, PO8, P8; IOT: PO3, PO7, P7).

Statistical analyses were separately carried on both responses (i.e., expression-specific and general visual). To quantify the overall magnitude of each response in a single value expressed in amplitude $(\mu \mathrm{V})$, individual FFT amplitude spectra were first baseline corrected by subtracting the mean surrounding noise (see above) and harmonics were summed until the $8^{\text {th }}$ harmonic for the general visual response, and until the $7^{\text {th }}$ harmonic for the expression-specific response excluding the one corresponding to the base frequency (i.e., $6 \mathrm{~Hz}$ ). Repeated-measures ANOVAs (i.e., one for each brain response) were conducted on these summed baseline-corrected amplitudes (BCA) with 
Expression (anger, disgust, fear, happiness, sadness and neutrality) and ROI ( $\mathrm{MO}, \mathrm{IOT}$, rOT) as withinsubject factors. Mauchly's test for sphericity violation was performed and Greenhouse-Geisser correction for degrees of freedom was applied whenever sphericity was violated. For significant effects, post-hoc comparisons were conducted using Tukey's HSD test. For visualization of both brain responses, signal-to-noise ratios (SNR) were calculated as summed uncorrected amplitudes divided by mean surrounding noise.

\subsection{Scalp topography analysis}

To assess whether the different facial expressions were associated with specific topographical patterns, we determined which electrodes across the whole scalp presented a significant expression-specific response on the grand-averaged uncorrected amplitudes summed across significant harmonics. Topographical differences were first normalized (McCarthy \& Wood, $1985)$ to equalize the scalp-wide global magnitude of the response across emotions, and Z-scores were then calculated for each electrode. A repeated-measures ANOVA was also conducted on normalized summed BCA with Expression (anger, disgust, fear, happiness, sadness and neutrality) and Electrode (64 channels) as within-subject factors to determine whether the different emotions elicit distinguishable topographical patterns when amplitude is equalized across them (Dzhelyova et al., 2017). We focused on the interaction between Expression and Electrode that would indicate different topographical distributions between facial expressions.

\subsection{Individual data analysis}

To assess the significance of the expression-specific response at an individual level, we also calculated Z-scores (see section 2.6) in every participant for summed uncorrected amplitudes over the electrodes belonging to the defined ROIs, and then for all the other electrodes.

\section{Results}

\subsection{Expression-specific response}

Since only one specific facial expression periodically appeared at $1.2 \mathrm{~Hz}$ (i.e., every $5^{\text {th }}$ stimulus) among all the other expressions randomly displayed at the $6 \mathrm{~Hz}$ base rate of stimulation, the brain response recorded at $1.2 \mathrm{~Hz}$ and its harmonics directly reflects single-glance categorization of a specific expression against the others regardless of expression-changes (captured in the general visual response elicited at the $6 \mathrm{~Hz}$ base rate). Visual inspection revealed that an expression-specific response was mainly recorded over lateral occipito-temporal and occipito-parietal regions, with 
larger amplitudes for fear and neutrality (Fig. 2). These two expressions elicited specific responses of high signal-to-noise ratio (SNR around 1.8, i.e. $80 \%$ of signal increase) suggesting that they are clearly discriminated when directly contrasted with all the other expressions. While the other facial expressions elicited weaker responses, they were nonetheless all visible on the EEG spectra (SNR around 1.4, i.e. $40 \%$ of signal increase) except for happiness (but with a significant nevertheless, see Z-scores below).

These observations from visual inspection were confirmed by the statistical analyses with a significant main effect of Expression $\left(F(2.6,36.6)=12.35, \varepsilon=0.52, \eta_{p}^{2}=0.477, p<.001\right)$, due to a larger expression-specific response for fear $(0.36 \pm 0.05$ (SEM) $\mu \mathrm{V})$ and neutrality $(0.37 \pm 0.08 \mu \mathrm{V})$ than for the other 4 expressions (anger: $0.09 \pm 0.03 \mu \mathrm{V}$, disgust: $0.14 \pm 0.03 \mu \mathrm{V}$, happiness: $0.06 \pm 0.03 \mu \mathrm{V}$, sadness: $0.12 \pm 0.03 \mu \mathrm{V}$, all $p s<.01$ ), and no other significant differences. This effect was qualified by an interaction between Expression and $R O I\left(F(10,140)=2.48, \eta_{p}^{2}=0.15, p=.009\right)$ with a larger response over $\mathrm{mO}(0.45 \pm 0.11 \mu \mathrm{V})$ than IOT $(0.25 \pm 0.06 \mu \mathrm{V})$ only for neutrality $(p=.001)$. However, further inspection of the spatial distribution of the response for each facial expression suggested variable topographies between expressions (Fig. 3).

To better identify the topographical patterns observed for each facial expression, we eliminated the large amplitude differences between expressions by normalizing the response amplitude across the whole scalp (McCarthy \& Wood, 1985; see Dzhelyova et al., 2017). As can be seen in the upper part of Figure 3, normalized summed baseline-corrected amplitudes (BCA) revealed a variable topography of the expression-specific response across the different expressions. Interestingly, while all facial expressions seem to elicit occipito-parietal activities, and present with a right hemisphere advantage, fear is the only expression showing a more ventral topographical distribution and a left hemisphere advantage. The repeated-measures ANOVA run on normalized summed BCA and including the 64 recording channels revealed a significant interaction between Expression and Electrode $\left(F(315,4410)=1.51, \eta_{p}^{2}=0.10, p<.001\right)$, confirming the variable spatial distributions of the expression-specific response across facial expressions when amplitude differences are controlled.

We also calculated Z-scores on normalized uncorrected amplitudes to determine which electrodes presented a significant expression-specific response for each emotion (bottom part of Figure 3). All facial expressions elicited a significant expression-specific response over at least 4 posterior electrodes. Responses to anger and sadness were centered over medial occipital sites while responses to disgust and happiness were sparser over posterior regions. The categorization of fear and neutrality elicited significant responses encompassing many posterior electrodes but with a greater right hemispheric response only for neutrality. Significant electrodes also confirmed that an 
expression-specific response was recorded over occipito-parietal channels for all facial expressions except for fear.

\subsection{Individual data analysis}

Individual data analyses revealed the strength of the expression-specific response and its reliability across participants. When combining all facial expressions, a significant expression-specific response was recorded for every participant over at least 2 out of the 14 electrodes included in the ROIs (Figure 4). When considering each facial expression separately, a significant expression-specific response was recorded over at least 2 electrodes over the scalp for every participant and for every facial expression. For fear in particular, the response was significant for every participant, with at least 2 out of the 14 electrodes included in the ROIs. The same was observed for neutrality, with at least 1 electrode included in the ROls, except for one participant who nevertheless displayed a significant response over another posterior electrode (P5). For disgust and happiness, 13 out of the 15 participants displayed a significant response over at least 1 electrode included in the ROls, the 2 other participants presenting a significant response elsewhere over the scalp. Finally, for anger and sadness, a significant response was observed over at least 1 electrode included in the ROls for 12 out of the 15 participants, the 3 other participants displaying a significant response elsewhere over the scalp.

\subsection{General visual response and behavioral data}

The brain response recorded at the base frequency and harmonics reflects the processes elicited by low- and high-level visual cues rapidly changing in the stream of stimulation (e.g., contrast, expression-change). In line with previous studies (Dzhelyova et al., 2017; Leleu et al., 2018), we found a clear medial occipital response of high amplitude (Figure 2). Accordingly, the main effect of $R O I$ $\left(F(2,28)=21.14, \eta_{p}^{2}=0.60, p<.001\right)$ confirmed the larger response over $\mathrm{mO}$ sites $(3.68 \pm 0.42 \mu \mathrm{V})$ than over the other ROIs ( $p s<.01$, IOT: $1.56 \pm 0.17 \mu \mathrm{V}$, rOT: $2.38 \pm 0.28 \mu \mathrm{V}$ ), with $48.3 \%$ of the overall signal recorded over mO regions (respectively $20.5 \%$ and $31.2 \%$ for IOT and rOT), the response at the rOT sites is also larger than over the IOT sites $(p<.05)$. Importantly, neither the main effect of Expression $(\mathrm{F}<1)$ nor its interaction with $R O I\left(\mathrm{~F}(10,140)=1.17, \eta_{\mathrm{p}}^{2}=0.07, p=.35\right)$ were significant, confirming that the facial expression displayed at the expression-specific frequency had no influence on the general visual response, and that the global attentional level that typically modulates such periodic brain activities (e.g., Kim et al., 2007) cannot account for the differences observed for the 
expression-specific response. In addition, behavioral data for the orthogonal task showed that the mean accuracy for detecting circle-to-square changes was $94.56 \%(S D=9.2)$, with a mean correct response time of $457 \mathrm{~ms}(S D=54)$. There were no differences between facial expressions for both accuracy and response times (both Fs $<1$ ).

\section{Discussion}

Here, we extend recent FPVS-EEG studies measuring facial expression discrimination (Dzhelyova et al., 2017; Leleu et al., 2018) by showing that when directly contrasted against all the other expressions, each facial expression elicits a specific signature over posterior brain areas in the EEG spectrum, thus providing evidence for specific neural processes dedicated to each facial expression. We further showed that rapid discrimination (i.e. at a glance) between facial expressions and assignment of one expression into a specific emotion category are subtended by discriminable activities at the brain level, with variable topographies between expressions. More specifically, fear and neutrality elicit a larger response than anger, disgust, sadness, and happiness, revealing a particular status for these two expressions among all the others. For fear, the response also differs qualitatively, with a more ventral (i.e., occipito-temporal) and left-hemispheric response than for the other expressions.

The main purpose of the study was to determine whether dissociable brain responses can be observed for the assignment of facial expressions into discrete emotional categories. In other words, our aim was to demonstrate that specific patterns of electrocortical activities can be recorded for each facial expression when neural activities elicited for every facial expression (e.g., a neural process that codes for facial movements in general) are eliminated. We controlled for those general neural responses using a frequency-tagging approach in which an expression-specific response was recorded at $1.2 \mathrm{~Hz}$ and harmonics by opposing a facial emotion to all the others, delineating the aspects of the response that are specifically elicited by that given facial expression. Indeed, since facial expressions were rapidly changing at the $6 \mathrm{~Hz}$ base rate of stimulation, neural activities reflecting the processing of any change of facial expression were captured by the general visual response recorded at $6 \mathrm{~Hz}$ and harmonics. To our knowledge, this is the first probing evidence of unique EEG signatures for each basic facial expression. Indeed, in previous studies, brain responses to variable facial expressions were generally confounded with other more general neural signals (e.g., face-related responses, expression-change responses) when measuring a large and global EEG response to the sudden onset of facial expression from a no-stimulus baseline as in typical ERP studies (Calvo \& Nummenmaa, 2016 for review), or a brain response to a change of expression from 
a neutral face as in recent FPVS-EEG studies (Dzhelyova et al., 2017; Leleu et al., 2018). Importantly, the different facial expressions did not modulate the general visual response elicited at $6 \mathrm{~Hz}$ and harmonics, and the behavioral data obtained for the orthogonal shape-change detection task, showing that the expression-specific responses are not accounted by attentional variations during visual stimulation but truly reflect the differential processing of facial expressions.

With the present design, we were thus able to reveal specific neural markers of high SNR (i.e., $\approx 1.4$ in average, $40 \%$ of signal increase compared with surrounding noise level) for each facial emotion in a few minutes of recording (i.e., only 3 minutes of stimulation were analyzed for each facial expression). These specific signatures were significant in every individual participant and presented with clear distinct topographies and amplitudes for the five basic expressions of happiness, anger, disgust, fear, and sadness, as well as for neutrality. While neutrality elicits a large response over right occipito-temporal sites, anger and sadness elicit more focal responses over medial occipital and parietal sites, predominantly over the right hemisphere for anger and more medial for sadness. Responses to disgust and happiness are more laterally distributed and encompass mostly central parietal regions, predominantly over the right hemisphere. In contrast, fear elicits a large response encompassing all occipito-temporal sites, predominantly over the left hemisphere. The EEG responses elicited with the present design may reflect the activity of brain regions involved in parting facial expressions into discrete categories, such as the STS (Said et al., 2010). The distinct topographies also suggest that neural activities originating from at least partially distinct brain regions are recorded, such as those from the extended network involved in facial emotion recognition (Calder \& Young, 2005; Haxby et al., 2000; Vytal \& Hamann, 2010). Indeed, many electrodes responded only to a subset of expressions, and none responded to all expressions. Thus, we propose that the present paradigm taps the activity of neural substrates specifically involved in the coding of discrete facial emotions, as those isolated by brain imaging studies (e.g., Vytal \& Hamann, 2010). These authors further underlined that the regions identified for each emotion category respond reliably to facial expressions, but also to other kinds of materials, visual or not. Thus, the expression-specific brain responses recorded here may also partly capture neural activities from areas that code for the emotional content of stimuli, irrespectively of their physical characteristics.

In this framework, both fear and neutrality appeared to elicit larger expression-specific responses. For neutrality, a face without emotional expressive features was opposed to all expressions of emotions. The observed large response for this non-emotional expression suggests that some brain regions respond to the general dissociation between non-emotional vs. emotional faces, that is, brain regions whose activity is related to the occurrence of (non-)expressive features, whatever their emotional content. In that respect, we observed a response pattern that was more 
typical of the right occipito-temporal activities usually reported for several face-related processes with the FPVS-EEG approach (e.g., Rossion, 2014a, 2014b). This suggests that the differential response to neutral faces originates from the core regions of the face-processing network (Calder \& Young, 2005; Haxby et al., 2000). Considering the nature of the design - which contrasted expressive and non-expressive faces - the specific response to neutrality may be mainly driven by cortical regions involved in the processing of changeable aspects of faces, notably the STS (e.g., Srinivasan, Golomb, \& Martinez, 2016). It may also reflect activity from the lateral fusiform gyrus, involved in the processing of invariant aspect of faces (Haxby et al., 2000), and also modulated by the emotional content of faces (Buchel \& Dolan, 2000; George, Driver, \& Dolan, 2001; Kawasaki et al., 2012; Morris et al., 1998; Vuilleumier, Armony, Driver, \& Dolan, 2001).

For fear, the response displayed the larger amplitude compared with the other emotions, with a more occipito-temporal topography and a left hemisphere advantage. This suggests that fear holds a special position as a facial expression, clearly discriminated from the other expressions, possibly in line with a selective "fear network" in the brain centered on the amygdala (Öhman \& Wiens, 2004). Accordingly, both neuroimaging (Vytal \& Hamann, 2010) and lesion studies (Adolphs, Tranel, Damasio, \& Damasio, 1994) have reliably associated fear with amygdala activity. It is unlikely that the response recorded in our study was elicited directly in the amygdala, considering the deep location of this structure. Rather, it could reflect the modulatory influence of this structure on the visual cortex. Such modulations were frequently suggested in brain imaging studies (Pessoa, McKenna, Gutierrez, \& Ungerleider, 2002; Vuilleumier, Armony, Driver, \& Dolan, 2001). Furthermore, such modulations were not observed in patients with amygdala damages (Vuilleumier, Richardson, Armony, Driver, \& Dolan, 2004). Thus, the stronger response we recorded for fear at posterior occipito-temporal sites, in comparison to all other emotional expressions, may reflect the reentrance modulation of the amygdala to the visual cortex (Kawasaki, Tsuchiya, Kovach, Nourski, Oya, Howard, \& Adolphs, 2012; Pourtois et al., 2010), or more generally the spreading of the response elicited by fear in the amygdala to occipito-temporal, STS, anterior temporal and orbitofrontal cortex (KrolakSalmon, Henaff, Vighetto, Bertrand, \& Mauguiere, 2004). Finally, it may reflect top-down influences from an amygdala-prefrontal system that increased monitoring of the environment to solve predictive uncertainly initiated by fearful expressions (see Whalen et al., 2013). In any event, thanks to FPVS-EEG, we were able to isolate a specific signature for fear compared with other facial expressions. It holds high promise for future research conducted on populations whose differential processing of fear can be impaired (e.g. individuals with autism spectrum disorders (ASD); Malaia, Cockerham, \& Rublein, 2017), in line with the successful recent application of FPVS-EEG in clinical research for testing e.g. face individuation in individuals with ASD (Vettori et al., 2018) or expressionchange perception in participants with 22q11.2 deletion syndrome (Leleu et al., 2019). 


\subsection{Limitations}

One limitation of the present study is the relatively small sample size that was not preexperimentally defined due to the exploratory nature of the study. However, thanks to the frequency-tagging approach which provides very high SNR, we were able to identify a significant expression-specific response in every participant. This suggests that this brain response to facial expression is reliable. Accordingly, power analysis conducted on BCA averaged across ROIs and facial expressions with a significance level $\alpha=.05$ (one-tailed, signal $>$ noise $=B C A>0$ ) revealed that with a mean amplitude of $0.19 \pm 0.11$ (SD) $\mu \mathrm{V}$, we obtained a large effect size of 1.73 (Cohen's d) leading to maximal power $1-\beta=1$. With this power, a similar effect could even be found with a smaller sample size $N=8$. Power would still be high $(1-\beta=0.83)$ if we consider a smaller effect size of $d=0.67$, corresponding to the lower limit of the $99 \%$ confidence interval calculated with the observed effect size. Hence, despite a small sample size, the brain response identified here seems to reflect a robust neural marker of the visual discrimination of facial expressions.

\section{Conclusions}

Taken together, the present findings demonstrate that specific EEG signatures can be isolated and quantified for different facial expressions of basic emotions using FPVS-EEG and direct contrasts between a given facial expression and all the others. These specific neural markers are of high SNR, reliably elicited in every individual participant in a few minutes of recording, and present with variable topographies, suggesting that different neural substrates underlie the brain coding of each facial expression of emotion. In this context, fear and neutrality elicit quantitatively and qualitatively different brain responses, suggesting particular status for neutral (i.e., no emotion) and fearful (i.e., threatened) faces in the human brain. Finally, the robustness of the present approach offers a unique opportunity to evaluate single-glance facial expression processing with precise issues on specific emotions and/or for various populations. 


\section{Acknowledgments}

The authors are grateful to Romain Patrux for help in data collection and to Renaud Brochard and the "Institut Universitaire de France" for assistance and financial support in performing EEG. This work received support from the "Conseil Régional Bourgogne FrancheComté" (PARI grant to FP, JYB and AL, and FABER grant to $A L$ ), the FEDER (European Funding for Regional Economic Development) and the French "Investissements d'Avenir" program, project ISITE-BFC (contract ANR-15-IDEX-03). MD is supported by the Fonds National de la Recherche Scientifique (FNRS). The authors declare no competing interest. 


\section{References}

Adolphs, R., Tranel, D., Damasio, H., Damasio, A. R., 1994. Impaired recognition of emotion in facial expressions following bilateral damage to the human amygdala. Nature 372 (6507), 669-672.

Adrian, E. D., Matthews, B. H. C., 1934. The Berger rhythm: potential changes from the occipital lobes in man. Brain 57 (4), 355-385.

Ashley, V., Vuilleumier, P., Swick, D., 2004. Time course and specificity of event-related potentials to emotional expressions. Neuroreport 15 (1), 211-216.

Barrett, L. F., Lindquist, K. A., Bliss-Moreau, E., Duncan, S., Gendron, M., Mize, J., Brennan, L., 2007. Of mice and men: natural kinds of emotions in the mammalian brain? A response to Panksepp and izard. Perspectives on Psychological Science, 2 (3), 297-312.

Barrett, L. F., Wager, T., 2006. The structure of emotion: Evidence from the neuroimaging studies. Current Directions in Psychological Science, 15 (2), 79-83.

Braddick, O. J., Wattam-Bell, J., Atkinson, J., 1986. Orientation-specific cortical responses develop in early infancy. Nature 320, 617-619.

Buchel, C., Dolan, R. J., 2000. Classical fear conditioning in functional neuroimaging. Current Opinion in Neurobiology, 10 (2), 219-223.

Calder, A. J., Young, A. W., 2005. Understanding the recognition of facial identity and facial expression. Nature Reviews Neuroscience 6, 641-651.

Calvo, M. G., Nummenmaa, L., 2016. Perceptual and affective mechanisms in facial expression recognition: an integrative review. Cognition and Emotion 30 (6), 1081-1106.

Calvo, M. G., Beltrán, D., 2013. Recognition advantage of happy faces: Tracing the neurocognitive processes. Neuropsychologia 51 (11), 2051-2060.

Calvo, M. G., Marrero, H., Beltrán, D., 2013. When does the brain distinguish between genuine and ambiguous smiles? An ERP study. Brain and Cognition 81 (2), 237-246.

Darwin, C. 1872. The expression of the emotions in man and animals. London, UK: John Murray. Retrieved from 〈http://darwin-online.org.uk〉.

Dzhelyova, M., Rossion, B., 2014a. Supra-additive contribution of shape and surface information to individual face discrimination as revealed by fast periodic visual stimulation. Journal of Vision $14(14,15), 1-14$.

Dzhelyova, M., Rossion, B., 2014b. The effect of parametric stimulus size variation on individual face discrimination indexed by fast period visual stimulation. BMC Neuroscience 15 (87), 1-12.

Dzhelyova, M., Jacques, C., Rossion, B., 2017. At a Single Glance: Fast Periodic Visual Stimulation Uncovers the Spatio-Temporal Dynamics of Brief Facial Expression Changes in the Human Brain. Cerebral Cortex 27 (8), 4106-4123.

Ekman, P., 1992. An argument for basic emotions. Cognition and Emotion 6 (3-4), 169-200.

Ekman, P., 1993. Facial expression and emotion. American Psychologist 48 (4), 384-392.

Ekman, P., 1994. Strong evidence for universals in facial expressions: a reply to Russell's mistaken critique. Psychological Bulletin 115 (2), 268-287.

Ekman, P., Friesen, W. V., 1971. Constants across cultures in the face and emotion. Journal of Personality and Social Psychology 17 (2), 124-129. 
Ekman, P., Friesen, W. V., Ellsworth, P., 1972. Emotion in the Human Face: Guidelines for Research and an Integration of Findings. New York: Pergamon Press.

Elfenbein, H. A., Ambady, N., 2002. On the universality and cultural specificity of emotion recognition: a meta-analysis. Psychological Bulletin 128 (2), 203-235.

Etcoff, N. L., Magee, J. J., 1992. Categorical perception of facial expressions. Cognition 44 (3), 227240.

George, N., Driver, J., Dolan, R. J., 2001. Seen gaze-direction modulates fusiform activity and its coupling with other brain areas during face processing. Neurolmage 13 (6), 1102-1112.

Harris, R. J., Young, A. W., Andrews, T. J., 2012. Morphing between expressions dissociates continuous from categorical representations of facial expression in the human brain. Proceedings of the National Academy of Sciences 109 (51), 21164-21169.

Harris, R. J., Young, A. W., Andrews, T. J., 2014. Dynamic stimuli demonstrate a categorical representation of facial expression in the amygdala. Neuropsychologia 56, 47-52.

Haxby, J. V., Hoffman, E. A., Gobbini, M. I., 2000. The distributed human neural system for face perception. Trends in Cognitive Sciences 4 (6), 223-233.

Kawasaki, H., Tsuchiya, N., Kovach, C. K., Nourski, K. V., Oya, H., Howard, M. A., Adolphs, R., 2012. Processing of facial emotion in the human fusiform gyrus. Journal of Cognitive Neuroscience 24 (6), 1358-1370.

Kim, Y. J., Grabowecky, M., Paller, K. A., Muthu, K., Suzuki, S., 2007. Attention induces synchronization-based response gain in steady-state visual evoked potentials. Nature Neuroscience 10 (1), 117-125.

Kim, H., Somerville, L., Johnstone, T., Polis, S., Alexander, A., Shin, L., Whalen, P., 2004. Contextual modulation of amygdala responsivity to surprised faces. Journal of Cognitive Neuroscience 16 (10), 1730-1745.

Krolak-Salmon, P., Henaff, M. A., Vighetto, A., Bertrand, O., Mauguiere, F., 2004. Early amygdala reaction to fear spreading in occipital, temporal, and frontal cortex: A depth electrode ERP study in human. Neuron 42 (4), 665-676.

Leleu, A., Dzhelyova, M., Rossion, B., Brochard, R., Durand, K., Schaal, B., Baudouin J.-Y., 2018. Tuning functions for automatic detection of brief changes of facial expression in the human brain. Neurolmage 179, 235-251.

Leleu, A., Favre, E., Yailian, A., Fumat, H., Klamm, J., Amado, I., Baudouin, J.-Y., Franck, N., Demily, C., 2019. An implicit and reliable neural measure quantifying impaired visual coding of facial expression: evidence from the 22q11.2 deletion syndrome. Translational Psychiatry 9, 67.

Leleu, A., Godard, O., Dollion, N., Durand, K., Schaal, B., Baudouin, J.-Y., 2015. Contextual odors modulate the visual processing of emotional facial expressions: An ERP study. Neuropsychologia 77, 366-379.

Leppänen, J. M., Kauppinen, P., Peltola, M. J., Hietanen, J. K., 2007. Differential electrocortical responses to increasing intensities of fearful and happy emotional expressions. Brain Research 1166, 103-109.

Liu-Shuang, J., Norcia, A. M., Rossion, B., 2014. An objective index of individual face discrimination in the right occipito-temporal cortex by means of fast periodic oddball stimulation.

Neuropsychologia 52, 57-72. 
Liu-Shuang, J., Torfs, K., Rossion, B., 2016. An objective electrophysiological marker of face individualisation impairment in acquired prosopagnosia with fast periodic visual stimulation. Neuropsychologia 83, 100-113.

Luo, W., Feng, W., He, W., Wang, N.-Y., Luo, Y.-J., 2010. Three stages of facial expression processing: ERP study with rapid serial visual presentation. Neurolmage 49 (2), 1857-1867.

Makeig, S., Bell, A. J., Jung, T.-P., Sejnowski, T.J., 1996. Independent component analysis of electroencephalographic data. In: D. Touretzky, M. Mozer \& M. Hasselmo (Eds.) Advances in Neural Information Processing Systems 8, 145-151. Cambridge, MA: MIT Press.

Malaia, E., Cockerham, D., Rublein, K., 2017, Visual integration of fear and anger emotional cues by children on the autism spectrum and neurotypical peers: An EEG study. Neuropsychologia (in press).

McCarthy, G., Wood, C. C., 1985. Scalp distributions of event-related potentials: An ambiguity associated with analysis of variance models. Electroencephalography and Clinical Neurophysiology 62 (3), 203-208.

Morris, J. S., Friston, K. J., Buchel, C., Frith, C. D., Young, A. W., Calder, A. J., et al., 1998. A neuromodulatory role for the human amygdala in processing emotional facial expressions. Brain 121, 47-57.

Norcia, A. M., Appelbaum, L. G., Ales, J. M., Cottereau, B. R., Rossion, B., 2015. The steady-state visual evoked potential in vision research: A review. Journal of Vision 15 (6): 4, 1-46.

Öhman, A., Wiens, S., 2004. The concept of an evolved fear module and cognitive theories of anxiety. In A. S. R. Manstead, N. Frijda \& A. Fischer (Eds.) Feelings and Emotions: The Amsterdam Symposium. Cambridge: Cambridge University Press.

Pessoa, L., McKenna, M., Gutierrez, E., Ungerleider, L. G., 2002. Neural processing of emotional faces requires attention. Proceedings of the National Academy of Sciences of the United States of America 99 (17), 11458-11463.

Pourtois, G., Spinelli, L., Seeck, M., Vuilleumier, P., 2010. Modulation of face processing by emotional expression and gaze direction during intracranial recordings in right fusiform cortex. Journal of cognitive neuroscience 22 (9), 2086-2107.

Puce, A., Syngeniotis, A., Thompson, J. C., Abbott, D. F., Wheaton, K. J., Castiello, U., 2003. The human temporal lobe integrates facial form and motion: evidence from fMRI and ERP studies. Neurolmage 19 (3), 861-869.

Regan, D., 1989. Human Brain Electrophysiology: Evoked Potentials and Evoked Magnetic Fields in Science and Medicine. Elsevier, New York.

Retter, T. L., Rossion, B., 2016. Uncovering the neural magnitude and spatio-temporal dynamics of natural image categorization in a fast visual stream. Neuropsychologia 91, 9-28.

Rossion, B., Torfs, K., Jacques, C., Liu-Shuang, J., 2015. Fast periodic presentation of natural images reveals a robust face-selective electrophysiological response in the human brain. Journal of Vision 15 (1): 18, 1-18.

Rossion, B., 2014a. Understanding face perception by means of human electrophysiology. Trends in Cognitive Sciences 18 (6), 310-318.

Rossion, B., 2014b. Understanding individual face discrimination by means of fast periodic visual stimulation. Experimental Brain Research 232 (6), 1599-1621.

Russell, J. A., 1980. A circumplex model of affect. Journal of Personality and Social Psychology 39, 1161-1178. 
Said, C. P., Moore, C. D., Engell, A. D., Todorov, A., Haxby, J. V., 2010. Distributed representations of dynamic facial expressions in the superior temporal sulcus. Journal of Vision 10 (5): 11, 1-12.

Sauter, D. A., Eisner, F., 2013. Commonalities outweigh differences in the communication of emotions across human cultures. Proceedings of the National Academy of Sciences of the United States of America 110 (3), E180.

Srinivasan, R., Golomb, J. D., Martinez, A. M., 2016. A neural basis of facial action recognition in humans. The Journal of Neuroscience. 36 (16), 4434-4442.

Tottenham, N., Tanaka, J. W., Leon, A. C., McCarry, T., Nurse, M., Hare, T. A., Nelson, C. A., 2009. The NimStim set of facial expressions: judgments from untrained research participants. Psychiatry Research 168 (3), 242-249.

Vettori, S., Dzhelyova, M., Van der Donck, S., Jacques, C., Steyaert, J., Rossion, B., Boets, B., 2018. Reduced neural sensitivity to rapid individual face discrimination in autism spectrum disorder. Neurolmage: Clinical (in press).

Vuilleumier, P., Pourtois, G., 2007. Distributed and interactive brain mechanisms during emotion face perception: evidence from functional neuroimaging. Neuropsychologia 45 (1), 174-194.

Vuilleumier, P., Armony, J. L., Driver, J., Dolan, R. J., 2001. Effects of attention and emotion on face processing in the human brain: An event-related fMRI study. Neuron 30 (3), 829-841.

Vuilleumier, P., Richardson, M. P., Armony, J. L., Driver, J., Dolan, R. J., 2004. Distant influences of amygdala lesion on visual cortical activation during emotional face processing. Nature Neuroscience 7 (11), 1271-1281.

Vytal, K., Hamann, S., 2010. Neuroimaging support for discrete neural correlates of basic emotions: A voxel-based meta-analysis. Journal of Cognitive Neuroscience 22 (12), 2864-2885.

Whalen, P. J., Raila, H., Bennett, R., Mattek, A., Brown, A., Taylor, J., et al., 2013. Neuroscience and facial expressions of emotion: The role of amygdala-prefrontal interactions. Emotion Review 5 (1), 78-83.

Williams, L. M., Palmer, D., Liddell, B. J., Song, L., Gordon, E., 2006. The 'when' and 'where' of perceiving signals of threat versus non-threat. Neurolmage 31 (1), 458-467.

Woodworth, R. S., Schlosberg, H., 1954. Experimental Psychology: Revised Edition. Henry Holt, New York. 


\section{Figure captions}

Figure 1. Stimuli and experimental design. Example of one individual female face with a neutral expression or expressing 5 basic emotions (anger, disgust, fear, happiness and sadness). Faces were presented through sinusoidal contrast variation and $10 \%$ randomized size variation with all emotions randomly displayed at a $6 \mathrm{~Hz}$ base rate ( 1 cycle $\approx 167 \mathrm{~ms}$ ) except for one which was periodically inserted at a lower rate of $1.2 \mathrm{~Hz}$ (every $5^{\text {th }}$ cycle $\approx 833 \mathrm{~ms}$ between each expression). This design thus isolates the specific response to a given emotion at the $1.2 \mathrm{~Hz}$ frequency regardless of expressionchanges occurring at the $6 \mathrm{~Hz}$ frequency.

Figure 2. Expression-specific and general visual response for each facial expression. A. Expressionspecific response for each facial expression. Left part: FFT signal-to-noise ratio (SNR) spectra for channels included in the regions-of-interest (ROIs) and for the 6 facial expressions. SNR for the expression-specific response are visible at the $1.2 \mathrm{~Hz}$ frequency and its harmonics (i.e., integer multiples, e.g., $2.4 \mathrm{~Hz}, 3.6 \mathrm{~Hz}$ ). Middle part: FFT signal-to-noise ratio (SNR) spectra calculated on the sum of significant harmonics of the $1.2 \mathrm{~Hz}$ expression-specific rate (F: until the 7th harmonic, i.e. 8.4 $\mathrm{Hz}$, excluding the $6 \mathrm{~Hz}$ base frequency) for channels included in the ROIs and for the 6 facial expressions. Note the high SNR for each facial expression except happiness, with large responses for fear and neutrality ( $S N R \approx 1.8,80 \%$ of signal increase) and weaker but still identifiable responses for the other expressions (SNR $\approx 1.4,40 \%$ of signal increase). Right part: 3D-topographical maps (posterior view) of summed baseline-corrected amplitudes (BCA) for the expression-specific response. B. General visual response elicited at the base rate. Left part: FFT signal-to-noise ratio (SNR) spectra calculated on the sum of significant harmonics of the $6 \mathrm{~Hz}$ base rate (F: until the 8th harmonic, i.e. $48 \mathrm{~Hz}$ ) for channels included in the regions-of-interest (ROIs) and for the 6 facial expressions. Note that SNR is high and does not differ between the 6 facial expressions (SNR $\approx 7,700$ $\%$ of single increase). Right part: 3D-topographical maps (posterior view) of summed baselinecorrected amplitudes (BCA) for the general visual response.

Figure 3. Scalp topography of the expression-specific response. Upper part: 3D-topographical maps (posterior view) of normalized summed baseline-corrected amplitudes (BCA) of the expressionspecific response for each facial expression. The scaling of the response equalizes global amplitude across expressions and reveals specific topographical patterns for each expression category. Bottom 
part: posterior electrodes with a significant $(Z>1.64, p<.05$, one-tailed, signal $>$ noise) response. For each expression, at least 4 electrodes were significant, and no electrode significantly responded to all expression categories. Note that neutrality and fear presented numerous significant electrodes. The response to fear was restricted to occipito-temporal channels compared with all other expressions.

Figure 4. Individual data: expression-specific response recorded for each participant. Left part: 3Dtopographical maps (posterior view) of the expression-specific response averaged across the 6 facial expressions (amplitude is scaled for each participant and individual maximum amplitudes are indicated above each map). Right part: 3D-topographical maps (posterior view) of the electrodes presenting a significant (i.e., $Z>1.64, p<.05$, one-tailed, signal $>$ noise) expression-specific response averaged across facial expressions for each individual participant. Electrodes in black are those included in the ROIs, those in gray are other posterior electrodes. 


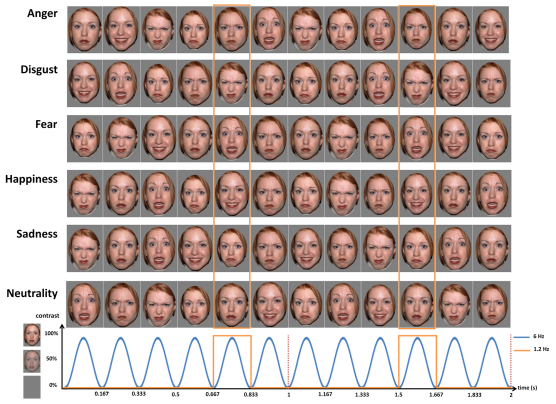


A

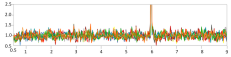

$$
25
$$$$
20
$$

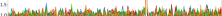

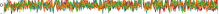

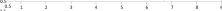
25
20
25

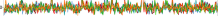

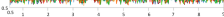
as. 29 15

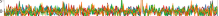

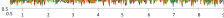
25 is

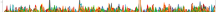

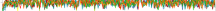

Expression-specific

$\langle 1.2 \mathrm{~Hz}+$ harmonics $\rangle$

SWR

14.

1.

ingophosing

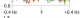

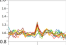

18
$64+5$

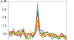

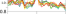

athe $+\quad 404 \mathrm{~m}$

i.

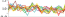

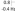

-isits ofis
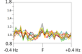

sit

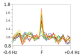

response

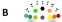

SNR
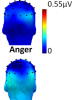

Disgust

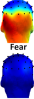

Happiness

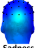

Sadness

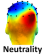

its.
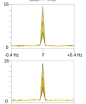

$u^{3+1}$
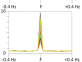

Q.

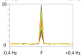

ain

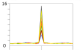

ante tou $\mathrm{Ex}$

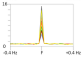

General visual

response

(6 $\mathrm{Hz}+$ harmonics)

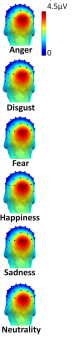




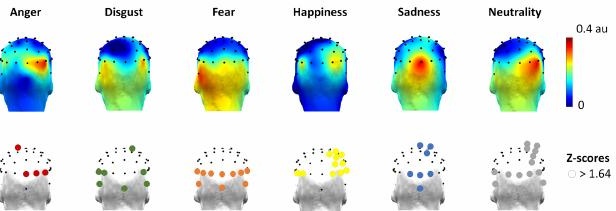




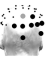

501

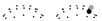

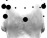

502

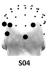

0.8

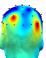

507

0.45

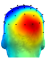

sa1

0.21

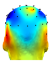

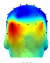

508

0.3

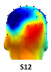

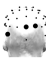

505

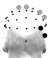

\$06

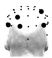

so7

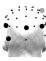

509
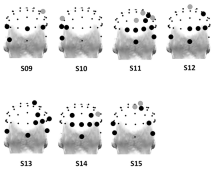

\section{Z-scores}

Electrodes within the ROIs e $>1.64$

Posterior electrodes e $>1.64$ 